\title{
Canaloplasty and Trabeculotomy Combined with Phacoemulsification in Open-Angle Glaucoma: Interim Results from the GEMINI Study
}

This article was published in the following Dove Press journal: Clinical Ophthalmology

\author{
Mark J Gallardo (iD) \\ Steven R Sarkisian Jr $\mathbb{( D}^{2}$ \\ Steven D Vold ${ }^{3}$ \\ Inder Paul Singh ${ }^{4}$ \\ Brian E Flowers ${ }^{5}$ \\ Anita Campbell ${ }^{6}$ \\ Kavita Dhamdhere ${ }^{7}$ \\ Thomas W Samuelson ${ }^{8}$ \\ On behalf of the GEMINI \\ study group \\ 'El Paso Eye Surgeons, PA, El Paso, TX, \\ USA; ${ }^{2}$ Oklahoma Eye Surgeons, PLLC, \\ Oklahoma City, OK, USA; ${ }^{3}$ Vold Vision, \\ Fayetteville, AR, USA; ${ }^{4}$ Eye Centers of \\ Racine \& Kenosha, Racine, WI, USA; \\ ${ }^{5}$ Ophthalmology Associates, Fort Worth, \\ TX, USA; ${ }^{6}$ Grene Vision Group, Wichita, \\ KS, USA; ${ }^{7}$ Sight Sciences, Menlo Park,

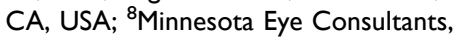 \\ Minneapolis, MN, USA
}

Correspondence: Kavita Dhamdhere Email kdhamdhere@sightsciences.com
Purpose: To report interim 6-month safety and efficacy outcomes of $360^{\circ}$ canaloplasty and $180^{\circ}$ trabeculotomy using the $\mathrm{OMNI}^{\circledR}$ Surgical System concomitantly with phacoemulsification in patients with open-angle glaucoma (OAG).

Setting: Fifteen multi-subspecialty ophthalmology practices and surgery centers located in 14 states (Alabama, Arizona, Arkansas, Florida, Georgia, Iowa, Kansas, Montana, Nebraska, North Dakota, Oklahoma, Pennsylvania, Texas, and Wisconsin).

Design: Prospective, multicenter, IRB approved study of patients treated with canaloplasty $\left(360^{\circ}\right)$ and trabeculotomy $\left(180^{\circ}\right)$. Eligible patients had cataract and mild-moderate OAG with intraocular pressure (IOP) $\leq 33 \mathrm{mmHg}$ on 1 to 4 hypotensive medications.

Methods: Medication washout prior to baseline diurnal IOP (Goldmann). Effectiveness outcomes included mean IOP and medications. Safety outcomes included adverse events (AE), best corrected visual acuity (BCVA) and secondary surgical interventions (SSI) Analysis includes descriptive statistics and t-tests evaluating change from baseline.

Results: A total of 137 patients were enrolled and treated. Mean diurnal IOP after washout was $23.8 \pm 3.1 \mathrm{mmHg}$ at baseline. At month 6,78\% (104/134) were medication free with IOP of $14.2 \mathrm{mmHg}$, a mean reduction of $9.0 \mathrm{mmHg}(38 \%) .100 \%(104 / 104)$ had a $\geq 20 \%$ reduction in IOP and $86 \%(89 / 104)$ had IOP $\geq 6$ and $\leq 18 \mathrm{mmHg}$. The mean number of medications at screening was $1.8 \pm 0.9$ and $0.6 \pm 1.0$ at month 6 . AE included transient hyphema $(4.6 \%)$ and IOP elevation $\geq 10 \mathrm{mmHg}(2 \%)$. There were no AE for loss of BCVA or recurring hyphema. There were no SSI.

Conclusion: Canaloplasty followed with trabeculotomy and performed concomitantly with phacoemulsification has favorable intra and perioperative safety, significantly reduces IOP and anti-glaucoma medications through 6 months in eyes with mild-moderate OAG.

Keywords: viscodilation, MIGS, open-angle glaucoma, glaucoma surgery, canaloplasty, trabeculotomy, OMNI

\section{Introduction}

The mechanisms of intraocular pressure (IOP) elevation in eyes with open-angle glaucoma $(\mathrm{OAG})$ are complex, and likely include disease-related alterations in the trabecular meshwork (TM), ${ }^{1,2}$ Schlemm's canal (SC), ${ }^{3}$ and the distal collector channels. ${ }^{4}$ Traditional surgeries - such as trabeculectomy and tube-shunt implantation bypass these structures and divert aqueous humor to the subconjunctival space. Subconjunctival filtering procedures are effective but are associated with significant short- and long-term complications, some of which can be vision-threatening., 5 
More recently, a growing family of minimally invasive glaucoma surgeries (MIGS) has evolved to achieve IOP reduction without bleb formation by restoring flow through the physiological conventional outflow pathway. MIGS procedures have the shared goal of balancing efficacy and safety, thereby expanding the indications for surgery to eyes with mild or moderate disease in which moderate IOP and/or medication reductions are desired at lower risk than with bleb-based procedures. ${ }^{7-10}$

MIGS procedures seek to increase conventional outflow by either facilitating aqueous passage through the TM and/or through SC. ${ }^{7-10}$ TM-based options include incisional and excisional goniotomy/trabeculotomy procedures as well as implantable microbypass stents to overcome the increased outflow resistance within the TM. Canal-based procedures such as the microcatheterization and transluminal viscodilation of up to 360 degrees of SC (canaloplasty), expand SC and collector channel ostia to facilitate aqueous egress from the eye. ${ }^{4,11}$ Outflow resistance can be considered as proximal (ie, juxtacanalicular TM and inner wall of SC) and distal (SC and the collector channels), with 50-70\% residing within the trabecular meshwork ${ }^{1,2}$ and the remaining $30-50 \%$ of outflow resistance within SC and collector channels. ${ }^{3,4}$ Addressing all sources of outflow resistance circumferentially would theoretically offer more consistent efficacy by treating all components of the conventional outflow pathway.

The OMNI ${ }^{\circledR}$ Surgical System (Sight Sciences) was designed to address all three sources of outflow resistance at the level of the TM, SC, and the collector channels. This handheld system features a cannula tip through which both a microcatheter and ophthalmic viscosurgical device (OVD) can be passed to sequentially perform viscodilation/canaloplasty and trabeculotomy/goniotomy; a gear wheel for deployment and retraction of the microcatheter; a reservoir for OVD; and a port for loading OVD into the handpiece. In studies published to date, titratable viscodilation/canaloplasty (up to $360^{\circ}$ ) followed by trabeculotomy (up to $360^{\circ}$ ) performed with this system has produced IOP reductions of $28 \%$ to $35 \%$ and medication reductions of $25-75 \%$ when deployed as a standalone procedure or in combination with phacoemulsification. ${ }^{12-15}$

This report provides early safety and effectiveness outcomes for $360^{\circ}$ viscodilation/canaloplasty followed by $180^{\circ}$ trabeculotomy with the OMNI system used concomitantly with phacoemulsification.

\section{Methods}

This was a prospective, multicenter, single-arm, clinical trial. The study protocol was reviewed and approved by a central IRB (Aspire, WCG group) and follows the tenets of the Declaration of Helsinki. All patients provided written informed consent to participate. The study was registered at ClinicalTrials.gov (NCT03861169), and patients were enrolled at fifteen US sites.

Eligible patients were adults age 22 years or older of either gender with visually significant cataract and mild to moderate open-angle glaucoma as defined by AAO/AGS/ ICD-10 Glaucoma Stage Definitions (including pigmentary and pseudoexfoliation glaucoma) with screening medicated IOP $\leq 33 \mathrm{mmHg}$ on $1-4$ topical IOP-lowering medications for at least 2 months prior to screening and unmedicated mean diurnal IOP (after appropriate washout) between 21 and $36 \mathrm{mmHg}$ and at least $3 \mathrm{mmHg}$ higher than the screening medicated IOP. Only one eye per patient could be enrolled. The eye with the higher IOP at baseline was selected as the study eye. If both were equal, the investigator selected the study eye. Patients were excluded if the anterior chamber angle was less than Schaffer grade 3 in all four quadrants by gonioscopy, if advanced glaucoma was present (mean deviation worse than $-12 \mathrm{~dB}$ ), if any form of glaucoma other than OAG was present, if laser trabeculoplasty had been performed within 3 months of the post-washout Baseline Visit, or if any prior glaucoma surgery (trabeculectomy, tube-shunt, or any MIGS procedure) had been performed at any time in the past. Patients deemed by investigators to be at risk of glaucoma progression due to medication washout or significant vision loss during the study period were excluded.

Potential patients underwent a screening evaluation that included comprehensive medical history and examination. Patients meeting screening criteria underwent washout of IOP-lowering medications (5 days for carbonic anhydrase inhibitors, 14 days for alpha adrenergic agonists, 28 days for all others) followed by diurnal IOP assessment at $9 \mathrm{am}, 12 \mathrm{pm}$ and $4 \mathrm{pm}$. Patients meeting final eligibility criteria then underwent surgery within 7 days.

Only eyes with uneventful standard phacoemulsification and intraocular lens implantation underwent the $360^{\circ}$ canaloplasty followed by $180^{\circ}$ trabeculotomy procedure. Eyes with complications related to cataract extraction or IOL implantation were exited from the study. The OMNI 
handpiece tip was inserted into the anterior chamber under intraoperative gonioscopy via the temporal clear corneal incision used for phacoemulsification and after the anterior chamber was deepened using OVD. Once advanced to the nasal angle, the canula tip was used to fashion a microgoniotomy incision in the TM. The flexible microcatheter was then advanced through the micro-goniotomy and around $180^{\circ}$ of $\mathrm{SC}$. The catheter was slowly retracted and a fixed volume of OVD was automatically dispensed to dilate SC and the collector channels. These steps were repeated along the remaining $180^{\circ}$ of SC through the same TM entry point in the opposite direction to complete the canaloplasty portion of the procedure. The microcatheter was then reinserted and re-advanced into the now-dilated $\mathrm{SC}$ and subsequently withdrawn slowly away from the TM entry point unroofing $\mathrm{SC}$ in a controlled manner to achieve $180^{\circ}$ of trabeculotomy. Approximately $70 \%$ of the trabeculotomies were made in the inferior hemisphere, $20 \%$ superior, $9 \%$ nasal, and 1\% temporal. The handpiece was withdrawn from the eye and the OVD was removed with standard irrigation/aspiration. A standard postoperative regimen of steroid, nonsteroidal anti-inflammatory drug and antibiotic was prescribed in every case.

Patients were examined at 1 day, 1 week, and 1, 3, and 6 months postoperatively. The study remains ongoing, and patients will be re-examined at 12 months postoperatively (with a terminal wash-out) for the final visit. Postoperative assessments included best-corrected visual acuity (BCVA), IOP, slit-lamp examination of the anterior segment, gonioscopy (beginning at week 1), and adverse event reporting. BCVA was assessed using the ETDRS chart along with manifest refraction at all time points except Day 1. IOP was assessed by Goldmann tonometry using operator/ reader system to avoid potential bias; the operator was responsible for operating the slit lamp and the instrument dial while the reader read and recorded the result without notifying the operator of the reading. At each time point, two measurements were taken, and the mean recorded. If the two readings differed by more than $2 \mathrm{mmHg}$, a third measurement was taken and the median recorded. Adverse events were identified by patient report, solicitation by study staff and by examination. The severity and causality of adverse events was assessed by investigator.

In the case that ocular hypotensive medication was required during the follow-up period to control IOP, the protocol was to re-introduce the same medication class (es) as used preoperatively with no more than one ocular hypotensive agent added at a single visit or within a 2 -week period. For subjects on multiple medications prior to the study and depending on a subject's pre-study medication regimen, the sequence of reintroduction was as follows: prostaglandin analogue, alpha agonist, beta blocker, Rho kinase inhibitor, carbonic anhydrase inhibitor.

In this interim analysis, the primary efficacy endpoints assessed were the reductions from screening to month 6 in mean medicated IOP for the full cohort, and the reduction from baseline mean diurnal unmedicated IOP among eyes medication-free at the follow-up visits. The proportion of eyes with $\geq 20 \%$ reduction in unmedicated IOP from baseline and the proportion of eyes with unmedicated IOP between 6 and $18 \mathrm{mmHg}$ inclusive were also assessed. Mean number of medications used and change from baseline are also reported for the full cohort. In addition, the 6-month outcome measures were stratified based on the number of hypotensive medications at the time of enrollment (screening). Safety endpoints included the nature and incidence of adverse events (AE), changes in BCVA from baseline, and any secondary surgical interventions (SSI).

Eligibility criteria for this study were modeled after the pivotal RCTs conducted and published on contemporary implantable MIGS devices (ie, Hydrus, iStent inject). The hypothesis and sample size of this trial are based on the data reported in the cataract extraction control arms of these trials. ${ }^{16,17}$ Phacoemulsification cataract surgery can result in some IOP reduction as was observed in these prior trials. The uniformity and high reproducibility of the post-phacoemulsification IOP and hypotensive medication use reported for the control arm in these trials provided sufficient information to serve as a historic-control to formulate a statistically sound hypothesis and systematic sample size calculation. Moreover, replication of the eligibility criteria, medication washout, and baseline IOP threshold for study entry from these prior studies will allow a reasonable estimate of the contribution of phacoemulsification to the observed IOP-lowering when the 12-month endpoint has been reached. Based on a one-sample $t$-test with a one-sided significance level of 0.025 (or two-sided $0.05)$, a sample size of 116 eyes provides a statistical power of $80 \%$ to detect a true mean IOP reduction of 7.5 $\mathrm{mmHg}$ or greater in OMNI-treated eyes. Accounting for attrition (estimated at 10\% over 12 months), a sample size of 130 patients was planned. For this interim analysis, effectiveness outcomes are constrained to descriptive statistics. 


\section{Results}

The study included 137 patients who met eligibility criteria. Three patients did not present for the 6-month visit due to the COVID19 restrictions and lockdown; therefore, six-month results are based on 134 patients. All patients went through appropriate washout prior to the treatment. This interim analysis included the full cohort of 137 eyes for the safety assessment and the subgroup of eyes that were on " 0 " medications at each of the timepoints for the effectiveness assessment. Most patients had a diagnosis of Primary OAG (93\%); $7 \%$ had Secondary OAG (Pseudoexfoliation - 6\%, Pigmentary $-1 \%$ ). Demographic data for the full cohort and consistent cohort are shown in Table 1.

Mean screening medicated IOP was $17.3 \pm 3.1 \mathrm{mmHg}$ and baseline unmedicated diurnal IOP was $23.8 \pm 3.1$ mmHg among the full cohort $(n=137)$. Mean screening medicated IOP was $17.2 \pm 3.1 \mathrm{mmHg}$ and baseline unmedicated diurnal IOP was $23.7 \pm 3.1 \mathrm{mmHg}$ for the medication-free cohort $(n=104)$. IOP outcome data for this interim analysis are presented in Table 2 and Figure 1. Among all eyes enrolled, mean IOP at Months $1(n=136)$, $3(n=132)$, and $6(n=134)$ ranged from $15.1-16.7 \mathrm{mmHg}$, and among the eyes on no medications at Months 1 $(\mathrm{n}=90), 3 \quad(\mathrm{n}=98)$, and $6(\mathrm{n}=104)$, IOP ranged from 14.2-15.6 $\mathrm{mmHg}$ (Figure 2) representing mean IOP reductions in these eyes of 7.6-9.0 mmHg (33-38\%). At Month

Table I Demographic Data $(n=\mid 37)$

\begin{tabular}{|l|c|}
\hline & Full Cohort, N=137 \\
\hline Age (yr), mean (SD) & $68.5(8.2)$ \\
\hline $\begin{array}{l}\text { Gender, } n(\%) \\
\text { Female } \\
\text { Male }\end{array}$ & $84(6 \mathrm{I})$ \\
\hline Race/Ethnicity, $\mathrm{n}(\%)$ & $53(39)$ \\
White & $112(82)$ \\
Other & $25(18)$ \\
\hline Study eye, $\mathrm{n}(\%)$ & $68(50)$ \\
Right & $69(50)$ \\
Left & \\
\hline Diagnosis $\mathrm{n},(\%)$ & $128(93.4)$ \\
POAG & $8(5.8)$ \\
\hline PXF & $\mathrm{I}(0.7)$ \\
\hline PG & $-3.7(3.6)$ \\
\hline Visual field MD (dB), mean (SD) & $3.7(2.6)$ \\
\hline Visual field PSD (dB), mean (SD) & \\
\hline
\end{tabular}

Abbreviation: SD, standard deviation.
$6,100 \%$ of the eyes requiring no medications $(104 / 104)$ achieved a $20 \%$ or greater IOP reduction, $86 \%$ of which had IOP between 6 and $18 \mathrm{mmHg}$.

The mean number of IOP-lowering medications used at screening in both the full and medication-free cohorts was $1.8 \pm 0.9$. Table 3 and Figure 3 provide medication outcome data for this analysis. At Months $1(\mathrm{n}=136), 3(\mathrm{n}=132)$, and 6 $(\mathrm{n}=134)$, the mean number of medications ranged from $0.4-0.6$, representing mean reductions of $69-81 \%$ from screening. The proportions of eyes on no medications at these time points ranged from $66-78 \%$, and all of these unmedicated eyes had IOP at all postoperative visits less than or equal to their preoperative medicated IOP. Thirtyfive patients who were on $>2$ medications at screening achieved an IOP reduction of $9.9 \pm 4.5 \mathrm{mmHg}(40 \%)$ at 6 months and $91 \%(32 / 35)$ of these patients achieved medication freedom. At 6 months $89 \%$ of patients (119/132) showed reduction in medications, $45 \%$ of which showed medication reduction by $>1$.

Adverse events through Month 6 are presented in Table 4. As is common with angle-based procedures, layered hyphema of $\geq 1 \mathrm{~mm}$ was the most common adverse event, occurring in 7 eyes $(4.6 \%)$, that resolved without further intervention or sequelae within four weeks of occurrence. Other AE included IOP increased $10 \mathrm{mmHg}$ or more above baseline at or after the Month 1 visit $(n=3,2.0 \%)$ and blepharitis $(n=2,1.3 \%)$. It is noteworthy that no AE for recurrent hyphema, loss in best corrected visual acuity, or for hypotony was reported. None of the AE was considered to be serious by the investigators.

By 6 months mean improvement in BCVA was 8.9 letters with $95 \%$ of patients having an improvement in BCVA. Through Month 6 there have been no SSI.

\section{Discussion}

The outcomes reported in the current study are consistent with prior reports evaluating the OMNI Surgical System. A retrospective analysis of 41 eyes of 24 patients with $\mathrm{OAG}$ undergoing the procedure in combination with phacoemulsification reported mean IOP reduction of $5.6 \mathrm{mmHg}$ (baseline not reported) overall and $9.6 \mathrm{mmHg}$ in eyes with baseline IOP $>22 \mathrm{mmHg}$ at four months postoperatively. ${ }^{12}$ A 12-month interim analysis of a planned 24-month prospective study of 17 eyes of 15 patients with mild to moderate OAG undergoing standalone ( 9 eyes) or combined ( 8 eyes) surgery with phacoemulsification found mean IOP reductions of $36 \%$ and medication reductions of $75 \%$ at 12 months postoperatively. ${ }^{13}$ A multicenter retrospective study reporting 
Table 2 Intraocular Pressure Outcomes

\begin{tabular}{|c|c|c|c|c|c|}
\hline & $\begin{array}{c}\text { Screening } \\
\text { Medicated, } \mathrm{N}=137\end{array}$ & $\begin{array}{c}\text { Baseline } \\
\text { Unmedicated, } N=137\end{array}$ & $\begin{array}{l}\text { Month I, } \\
\mathrm{N}=136\end{array}$ & $\begin{array}{l}\text { Month 3, } \\
\mathrm{N}=132\end{array}$ & $\begin{array}{c}\text { Month 6, } \\
\mathrm{N}=134\end{array}$ \\
\hline Mean (SD) IOP, mmHg & \multirow[t]{7}{*}{$17.3(3.1)$} & \multirow[t]{7}{*}{$23.8(3.1)$} & $16.7(6.6)$ & $15.2(3.6)$ & $15.1(3.9)$ \\
\hline No. of eyes on 0 medications & & & $90(66 \%)$ & $98(74 \%)$ & $104(78 \%)$ \\
\hline Mean (SD) IOP, $\mathrm{mmHg}^{\mathrm{a}}$ & & & $15.6(5.2)$ & $14.5(2.9)$ & $14.2(3.1)$ \\
\hline Mean (SD) IOP reduction from unmedicated baseline ${ }^{a}$ & & & $7.6(4.6)$ & $8.7(3.3)$ & $9.0(3.3)$ \\
\hline Mean (SD) percent IOP reduction from unmedicated baseline & & & $33(19)$ & $37(13)$ & $38(13)$ \\
\hline No. of eyes with IOP reduction $\geq 20 \%^{a}$ & & & $74(82 \%)$ & $97(99 \%)$ & $104(100 \%)$ \\
\hline $\begin{array}{l}\text { No. of eyes (\%) with IOP reduction } \geq 20 \% \text { and IOP }>6 \\
\mathrm{mmHg} \text { and } \leq 18 \mathrm{mmHg}^{\mathrm{a}}\end{array}$ & & & $65(72 \%)$ & $90(92 \%)$ & $89(86 \%)$ \\
\hline
\end{tabular}

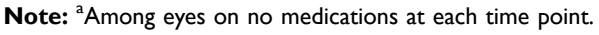

Abbreviations: IOP, intraocular pressure; SD, standard deviation.

12-month outcomes showed IOP decreasing from 21.9 to 15.1 $\mathrm{mmHg}$ with a $45 \%$ reduction in medications (combined with phaco cohort, baseline IOP $>18 \mathrm{mmHg}$ ) and from 21.8 to 15.6 $\mathrm{mmHg}$ with a $29 \%$ reduction in medications (standalone cohort, baseline IOP > $18 \mathrm{mmHg})^{14,15}$

Conceptually, sequential canaloplasty and trabeculotomy procedure lowers IOP by addressing three separate sites of outflow resistance. The juxtacanalicular TM and inner wall of SC are the site of at least $50 \%$ of resistance to aqueous outflow in eyes with OAG. ${ }^{1,2} \mathrm{Ab}$ interno trabeculotomy effectively lowers IOP by removing this barrier to aqueous outflow. Studies of gonioscopy-assisted transluminal trabeculotomy (GATT) - which can be performed with either a suture or microcatheter-have reported IOP reductions on the order of $30-45 \%$ and medication reductions of approximately $30-70 \%$ as a standalone procedure; when performed in combination with phacoemulsification, IOP reductions of approximately $30-70 \%$ and medication reductions of approximately $45-90 \%$ have been reported through 6-24 months of follow-up. ${ }^{18-25} \mathrm{Ab}$ interno trabeculotomy using the TRAB360 device lowered IOP approximately $30 \%$ and

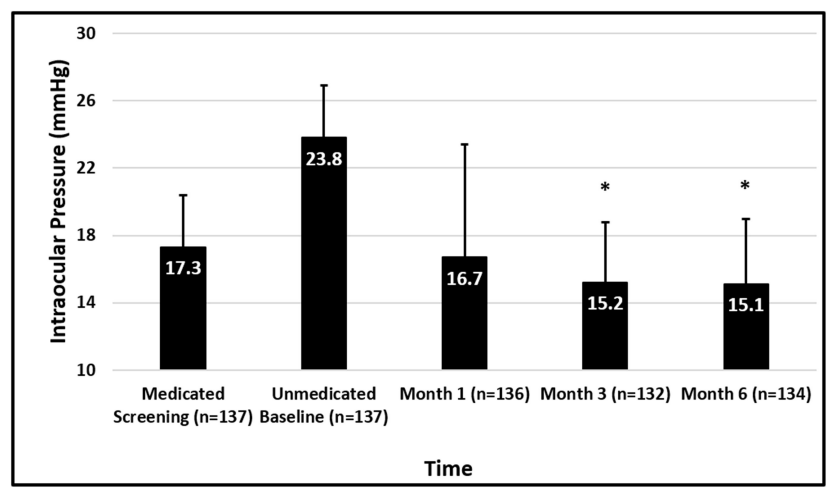

Figure I Mean IOP at each time point for the full cohort $(n=137)$. Error bars represent standard deviation. *Indicates $\mathrm{P}<0.0001$. medications approximately $35-80 \%$ in a retrospective study of patients with refractory glaucoma of various etiologies. ${ }^{26}$

In the present study, the protocol specified that trabeculotomy would be limited to 180 degrees. This standard was mandated in an effort to limit inter-site variability which could have complicated data interpretation. It is therefore not known if a complete 360 degree trabeculotomy would provide additional efficacy. Location of the trabeculotomy was not however dictated by the protocol but instead was left to surgeon discretion. The fact that a combined $90 \%$ were performed inferiorly and superiorly likely reflects two influences; the standard MIGS procedure of accessing the anterior chamber through a temporal clear corneal incision with the consequent nasal equatorial access to Schlemm's canal, and also by "handedness" of the surgeon (right or left handed) along with which hemisphere had been viscodilated first. The authors can only speculate as to why $9 \%$ were carried out for the nasal hemisphere and $1 \%$ temporally. It could be that in some cases the angle was maximally open with the most optimum visualization in these areas. However, the data

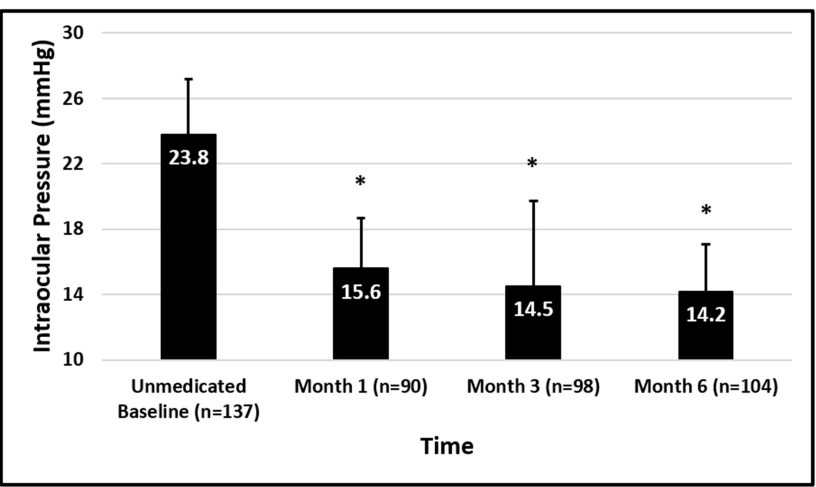

Figure 2 Mean IOP at each time point among patients on no medications at each time point. Error bars represent standard deviation. *Indicates $\mathrm{P}<0.0001$. 
Table 3 Medication Use Outcomes

\begin{tabular}{|l|c|c|c|c|}
\hline & Screening Medicated, N=137 & Month I, N=136 & Month 3, N=132 & Month 6, N=134 \\
\hline Mean (SD) no. of anti-glaucoma medications & $1.8(0.9)$ & $0.6(0.9)$ & $0.4(0.8)$ & $0.6(1.0)$ \\
Mean (SD) [\%] reduction from Screening & & $1.2(1.2)[69 \%]$ & $1.4(1.1)[79 \%]$ & $1.5(1.1)[81 \%]$ \\
No. of eyes (\%) on 0 medications and no increase in IOP & & $87(64 \%)$ & $97(73 \%)$ & $104(78 \%)$ \\
No. of eyes (\%) with no increase in medications & & $109(80 \%)$ & $117(89 \%)$ & $119(89 \%)$ \\
\hline
\end{tabular}

Abbreviations: IOP, intraocular pressure; SD, standard deviation.

collected does not allow knowing with certainty. It is possible that the location of the trabeculotomy could have some influence on effectiveness. There is limited published data available bearing on this question. The work of Huang and others using angiography and OCT indicate that there is little difference between superior and inferior quadrants in activity of outflow channels although there is some evidence that there is greater activity nasally than temporally. ${ }^{27}$ What is less clear is whether maximum efficacy would be achieved by targeting a quadrant that already has active flow (and therefore may not require treatment) or by treatment of an area of low activity. As both inferior and superior hemisphere treatment equally include half of the nasal and temporal hemispheres, we cannot address the question with the present dataset; however, it is an interesting and important question that should be studied.

Schlemm's canal and the distal collector channels also contribute to the resistance of aqueous humor outflow in glaucomatous eyes. Compared to eyes without glaucoma, $\mathrm{SC}$ in eyes with POAG is approximately 50\% narrowed, with subsequent $50 \%$ reduction in its outflow facility. ${ }^{3}$ In the setting of elevated IOP, herniation of the inner wall of $\mathrm{SC}$ into the openings to the collector channels may further reduce outflow. ${ }^{4}$ Bench studies using perfused human anterior segments have demonstrated that dilation of

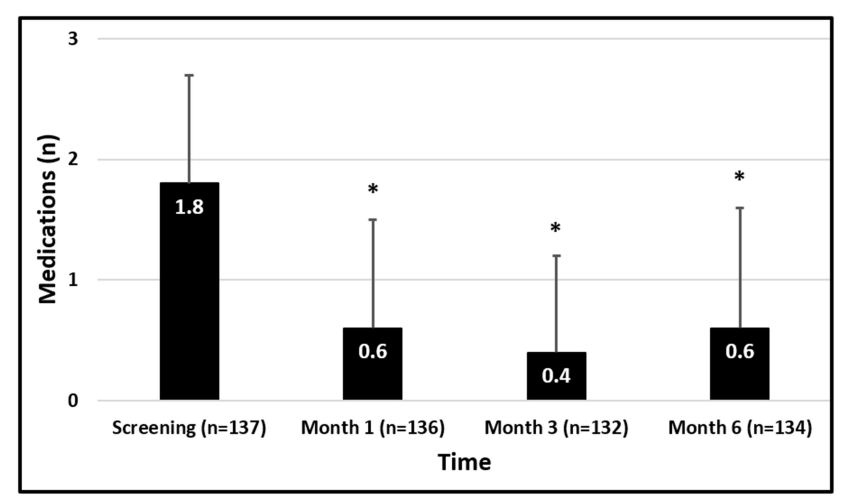

Figure 3 Ocular hypotensive medication use. Error bars represent standard deviation. *Indicates $\mathrm{P}<0.000 \mathrm{I}$. a 3-clock hour arc of SC with a scaffold results in a $79 \%$ increase in outflow facility or a $40 \%$ reduction in outflow resistance (the reciprocal of facility). ${ }^{28}$ Micrographs taken following viscodilation of Schlemm's canal in primate and cadaver eyes show an expanded lumen, widely dilated collector channel ostia and disruption of the inner wall of SC. ${ }^{11}$ The canaloplasty component of the OMNI dual procedure dilates the canal and re-establishes collector channel patency, both of which contribute to IOP reductions. ${ }^{4,11}$ In studies of canaloplasty, IOP reductions of $30-41 \%$ and medication reductions of up to $89 \%$ have been reported. ${ }^{29-31}$

In our study mean IOP decreased a modest amount between Month 1 and Months 3 and 6 while the proportion of patients medication-free showed a corresponding increase suggesting that effectiveness may increase with time. While we cannot determine the reason for this with certainty, we can speculate that the trends are related to standard post-op steroid use. It is known that $\sim 90 \%$ of glaucoma patients are steroid responders. ${ }^{32,33}$ As topical steroids are de rigueur following cataract or MIGS surgery for the first 30 days, it stands to reason that there will be some effect on mean IOP over this interval and that in some instances, surgeons will prophylactically (or reactively) provide concomitant treatment with topical ocular hypotensive medication. As the steroid is discontinued (generally) beyond 1 month, the IOP steroid response will wane as will the need for the IOP-lowering drops.

Three patients experienced an IOP increase $\geq 10 \mathrm{mmHg}$ above the baseline at $\geq 1$ month postoperatively. These were managed by addition of a topical prostaglandin ana$\log$ and (in one case) discontinuation of topical steroid. Subsequent visual fields did not suggest progression (MD within $\sim 1.5 \mathrm{~dB}$ of baseline values) and fundus examination showed that cup to disc ratio remained unchanged. IOP was controlled at the follow-up IOP check and all later study visits.

This study's design has several important strengths relative to much of the MIGS-related literature. For example, outside of PMA registration trials, few MIGS studies 
Table 4 The Nature and Incidence of Adverse Events

\begin{tabular}{|l|l|l|l|l|}
\hline & N (\%) & Serious ${ }^{\text {a }}$ & Device Related & Status \\
\hline Layered hyphema $\geq 1 \mathrm{~mm}$ & $7(4.6 \%)$ & No & Yes & Recovered spontaneously without sequel \\
IOP increase $\geq 10 \mathrm{mmHg}$ above baseline at $\geq I$ month & $3(2.0 \%)$ & No & Yes & Recovered without sequel with hypotensive medication added \\
Blepharitis & $2(1.3 \%)$ & No & No & Recovered spontaneously without sequel \\
Clinically significant cystoid macular edema & $I(0.7 \%)$ & No & No & Ongoing, on topical steroid \\
Internal hordeolum & $I(0.7 \%)$ & No & No & Ongoing with treatment \\
Keratoconjunctivitis sicca & $I(0.7 \%)$ & No & No & Ongoing with treatment \\
Vitreous hemorrhage & $I(0.7 \%)$ & No & No & Recovered spontaneously without sequel \\
\hline
\end{tabular}

Notes: ${ }^{a} \mathrm{An}$ adverse event that results in death, is life-threatening, requires in-patient hospitalization or prolongs existing hospitalization, necessitates medical or surgical intervention to preclude permanent impairment of a body function or permanent damage to a body structure, or is sight threatening.

Abbreviation: IOP, intraocular pressure.

have included evaluations of change in unmedicated IOP. This study's protocol incorporates a washout at baseline and at Month 12 (to be reported in subsequent paper) to facilitate such an analysis. To approximate this outcome at interim visits (when no washout was implemented) we conducted an analysis including only eyes on no medications at each time point. While this could introduce a bias in that only "responders" (ie, medication-free) were included, we also report separately on all enrolled eyes and the overall congruence of the results argues against significant bias. The prospective design also sets this study apart from the most MIGS publications. A weakness of the study is the reference to a historic control group, although this has been mitigated by powering the study to detect IOP changes in excess of those expected from phacoemulsification alone using the highly uniform data within the major randomized, controlled MIGS trials. ${ }^{17,18}$ Moreover, similarity in the baseline characteristics (age, gender, ethnicity, glaucoma severity) of the patients in this study with the historic control arms further validates the basis of using published literature to estimate the additive effect of combined $360^{\circ}$ canaloplasty and $180^{\circ}$ trabeculotomy to phacoemulsification $(-5.4$ and $-5.3 \mathrm{mmHg}$ for phacoemulsification only control groups at 24 months in iStent and Hydrus studies). ${ }^{16,17}$

Several papers suggest that canaloplasty (both ab interno and ab externo) and canaloplasty combined with trabeculotomy can have substantial IOP-lowering efficacy in patients with OAG (see ref 34 and references therein). ${ }^{34}$ However, the present work is one of very few prospective trials and the only prospective study of concomitant canaloplasty and trabeculotomy. A contribution of elements study looking at IOP lowering for the OMNI component procedures would be interesting and could shed light on the relative importance of distal and proximal outflow resistance. There are no such studies to our knowledge at present. In summary, this interim analysis of a planned 12 -month study demonstrates that $360^{\circ}$ ab interno canaloplasty and $180^{\circ}$ ab interno trabeculotomy, performed with the OMNI Surgical System used concomitantly with phacoemulsification in eyes with open-angle glaucoma safely produced clinically significant reductions in both IOP and the need for IOP-lowering medications through the initial 6 months post-operative period. The potential to address all three points of resistance using one minimally invasive surgical approach by combining ab interno viscodilation/canaloplasty and trabeculotomy/goniotomy is unique to this device. The safety profile of this procedure is highly favorable, with few adverse events, none of which were serious. Likewise, treatment-related adverse events resolved with minimal or no intervention. This procedure should be considered in eyes with mild to moderate OAG in which reductions in IOP and/or IOPlowering medications are desired. Full 12-month results of the entire cohort will be reported upon study completion.

\section{Data Sharing Statement}

The authors do not intend to share participant-level data. Other queries or requests should be directed to the corresponding author $(\mathrm{KD})$.

\section{Acknowledgments}

Dr. Tony Realini (Hypotony Holdings, LLC) prepared an initial draft manuscript. Jaime Dickerson, $\mathrm{PhD}$ is acknowledged for final revisions and editorial assistance. GEMINI study group: Alex Cohen, Michael Greenwood, Sebastian Heersink, Arkadiy Yadgarov, Lorne Schlecht, Mark Pyfer, Anil Vedula, Anita Campbell, Brandon Baartman, Russell Swan, and Yi-Jing Duh.

\section{Disclosure}

SRS Jr and SDV are consultants and speakers for Sight Sciences. SRS Jr reports grants, Allergan, Glaukos, BVI, 
Santen, Bausch and Lomb, Aerie, Alcon, Ocular Science, and Katena, outside the submitted work. SDV reports grants from Glaukos, Ivantis, Allergan, and iStar Medical, outside the submitted work. BEF reports personal fees from Sight Science, during the conduct of the study; grants, personal fees from Alcon, iStar Medical, Ivantis, Glaukos; grants from Aerie Pharmaceuticals, Nicox, and Santen; personal fees from Bausch and Lomb and New World Medical, outside the submitted work. TWS reports personal fees from Sight Science, Glaukos, Ivantis, New World Medical, Santen, Allergan, Alcon Surgical, and MicroOptix, during the conduct of the study; personal fees from Sight Science, outside the submitted work. All other authors are speakers for Sight Sciences. KD is an employee of Sight Sciences, Inc. The authors report no other conflicts of interest in this work.

\section{References}

1. Grant WM. Experimental aqueous perfusion in enucleated human eyes. Arch Ophthalmol. 1963;69:783-801. doi:10.1001/ archopht.1963.00960040789022

2. Maepea O, Bill A. Pressures in the juxtacanalicular tissue and Schlemm's canal in monkeys. Exp Eye Res. 1992;54:879-883.

3. Allingham RR, de Kater AW, Ethier CR. Schlemm's canal and primary open angle glaucoma: correlation between Schlemm's canal dimensions and outflow facility. Exp Eye Res. 1996;62:101-109. doi:10.1006/exer.1996.0012

4. Battista SA, Lu Z, Hofmann S, et al. Reduction of the available area for aqueous humor outflow and increase in meshwork herniations into collector channels following acute IOP elevation in bovine eyes. Invest Ophthalmol Vis Sci. 2008;49:5346-5352. doi:10.1167/iovs.081707

5. Gedde SJ, Schiffman JC, Feuer WJ, et al. Treatment outcomes in the Tube Versus Trabeculectomy (TVT) study after five years of follow-up. Am J Ophthalmol. 2012;153:789-803 e2. doi:10.1016/j. ajo.2011.10.026

6. Gedde SJ, Herndon LW, Brandt JD, et al. Postoperative complications in the Tube Versus Trabeculectomy (TVT) study during five years of follow-up. Am $J$ Ophthalmol. 2012;153:804-814. doi:10.1016/j.ajo.2011.10.024

7. Richter GM, Coleman AL. Minimally invasive glaucoma surgery: current status and future prospects. Clin Ophthalmol. 2016;10:189-206. doi:10.2147/OPTH.S80490

8. Lavia C, Dallorto L, Maule M, et al. Minimally-invasive glaucoma surgeries (MIGS) for open angle glaucoma: a systematic review and meta-analysis. PLoS One. 2017;12:e0183142. doi:10.1371/journal. pone. 0183142

9. Pillunat LE, Erb C, Junemann AG, Kimmich F. Micro-invasive glaucoma surgery (MIGS): a review of surgical procedures using stents. Clin Ophthalmol. 2017;11:1583-1600. doi:10.2147/OPTH. S135316

10. Chen DZ, Sng CCA. Safety and efficacy of microinvasive glaucoma surgery. $J$ Ophthalmol. 2017;2017:3182935. doi:10.1155/2017/ 3182935

11. Smit BA, Johnstone MA. Effects of viscoelastic injection into Schlemm's canal in primate and human eyes: potential relevance to viscocanalostomy. Ophthalmology. 2002;109:786-792. doi:10.1016/ S0161-6420(01)01006-5
12. Brown RH, Tsegaw S, Dhamdhere K, Lynch MG. Viscodilation of Schlemm canal and trabeculotomy combined with cataract surgery for reducing intraocular pressure in open-angle glaucoma. $J$ Cataract Refract Surg. 2020;46:644-645. doi:10.1097/j.jcrs.0000000000000107

13. Grabska-Liberek I, Duda P, Rogowska M, et al. 12-month interim results of a prospective study of patients with mild to moderate openangle glaucoma undergoing combined viscodilation of Schlemm's canal and collector channels and $360^{\circ}$ trabeculotomy as a standalone procedure or combined with cataract surgery. Eur J Ophthalmol. 2020.

14. Vold S, Williamson BK, Hirsch L, et al. Canaloplasty and trabeculotomy with the OMNI system in pseudophakic patients with open-angle glaucoma: the ROMEO study. Ophthalmol Glaucoma. 2020;S2589-4196(20)30264-7. doi:10.1016/j.ogla.2020.10.001

15. Hirsch L, Cotliar J, Vold S, et al. Canaloplasty and trabeculotomy ab interno with the OMNI system combined with cataract surgery in open-angle glaucoma: 12-month outcomes from the ROMEO study. J Cataract Refract Surg. 2020. doi:10.1097/j.jcrs.0000000000000552

16. Samuelson TW, Sarkisian SR, Lubeck DM, et al. Prospective, randomized, controlled pivotal trial of an ab interno implanted trabecular micro-bypass in primary open-angle glaucoma and cataract. Ophthalmology. 2019;126:811-821. doi:10.1016/j.ophtha.2019.03.006

17. Samuelson TW, Chang DF, Marquis R, et al. A Schlemm canal microstent for intraocular pressure reduction in primary open-angle glaucoma and cataract: the HORIZON study. Ophthalmology. 2019;126:29-37. doi:10.1016/j.ophtha.2018.05.012

18. Grover DS, Godfrey DG, Smith O, et al. Gonioscopy-assisted transluminal trabeculotomy, ab interno trabeculotomy: technique report and preliminary results. Ophthalmology 2014;121:855-861.

19. Grover DS, Godfrey DG, Smith O, et al. Outcomes of gonioscopy-assisted transluminal trabeculotomy (GATT) in eyes with prior incisional glaucoma surgery. $J$ Glaucoma. 2017;26:41-45. doi:10.1097/IJG.0000000000000564

20. Rahmatnejad K, Pruzan NL, Amanullah S, et al. Surgical outcomes of gonioscopy-assisted transluminal trabeculotomy (GATT) in patients with open-angle glaucoma. $J$ Glaucoma. 2017;26:1137-1143. doi:10.1097/IJG.0000000000000802

21. Grover DS, Smith O, Fellman RL, et al. Gonioscopy-assisted transluminal trabeculotomy: an ab interno circumferential trabeculotomy: 24 months follow-up. J Glaucoma. 2018;27:393-401. doi:10.1097/ IJG.0000000000000956

22. Aktas Z, Ozmen MC, Atalay HT, Ucgul AY. Evaluation of episcleral venous fluid wave during gonioscopy assisted transluminal trabeculotomy in patients with advanced glaucoma. Eye. 2019;33:668-673. doi:10.1038/s41433-018-0254-5

23. Olgun A, Aktas Z, Ucgul AY. XEN gel implant versus gonioscopy-assisted transluminal trabeculotomy for the treatment of open-angle glaucoma. Int Ophthalmol. 2020;40:1085-1093. doi:10.1007/s10792-019-01271-w

24. Baykara M, Poroy C, Erseven C. Surgical outcomes of combined gonioscopy-assisted transluminal trabeculotomy and cataract surgery. Ind J Ophthalmol. 2019;67:505-508. doi:10.4103/ijo.IJO_1007_18

25. Hirabayashi MT, Lee D, King JT, et al. Comparison of surgical outcomes of $360^{\circ}$ circumferential trabeculotomy versus sectoral excisional goniotomy with the Kahook dual blade at 6 months. Clin Ophthalmol. 2019;13:2017-2024. doi:10.2147/OPTH.S208468

26. Sarkisian SR, Mathews B, Ding K, et al. 360 degrees ab-interno trabeculotomy in refractory primary open-angle glaucoma. Clin Ophthalmol. 2019;13:161-168. doi:10.2147/OPTH.S189260

27. Huang AS, Penteado RC, Saha SK, et al. Fluorescein aqueous angiography in live normal human eyes. J Glaucoma. 2018;27:957-964. doi:10.1097/IJG.0000000000001042

28. Toris CB, Pattabiraman PP, Tye G, et al. Outflow facility effects of 3 Schlemm's canal microinvasive glaucoma surgery devices. Ophthalmol Glaucoma. 2020;3:114=121. doi:10.1016/j. ogla.2019.11.013 
29. Ondrejka S, Körber N. $360^{\circ}$ ab-interno Schlemm's canal viscodilation in primary open-angle glaucoma. Clin Ophthalmol. 2019;13:1235-1246. doi:10.2147/OPTH.S203917

30. Davids AM, Pahlitzsch M, Boeker A, et al. Ab interno canaloplasty (ABiC)-12-month results of a new minimally invasive glaucoma surgery (MIGS). Graefes Arch Clin Exp Ophthalmol. 2019;257:1947-1953. doi:10.1007/s00417-019-04366-3

31. Gallardo MJ, Supnet RA, Ahmed IK. Viscodilation of Schlemm's canal for the reduction of IOP via an ab-interno approach. Clin Ophthalmol. 2018;12:2149-2155. doi:10.2147/OPTH.S177597
32. Armaly MF. Effect of corticosteroids on intraocular pressure and fluid dynamics II. Effect of dexamethasone in the glaucomatous eye. Arch Ophthalmol. 1963;70(4):492-499. doi:10.1001/ archopht.1963.00960050494011

33. Becker B. Intraocular pressure response to topical corticosteroids. Invest Ophthalmol. 1965;26:198-205.

34. Dickerson JE Jr, Brown RH. Circumferential canal surgery: a brief history. Curr Opin Ophthalmol. 2020;31:139-146. doi:10.1097/ ICU.0000000000000639

\section{Publish your work in this journal}

Clinical Ophthalmology is an international, peer-reviewed journal covering all subspecialties within ophthalmology. Key topics include: Optometry; Visual science; Pharmacology and drug therapy in eye diseases; Basic Sciences; Primary and Secondary eye care; Patient Safety and Quality of Care Improvements. This journal is indexed on PubMed

Submit your manuscript here: https://www.dovepress.com/clinical-ophthalmology-journal
Central and CAS, and is the official journal of The Society of Clinical Ophthalmology (SCO). The manuscript management system is completely online and includes a very quick and fair peer-review system, which is all easy to use. Visit http://www.dovepress.com/ testimonials.php to read real quotes from published authors. 